\title{
Esquizofrenia y funcionamiento: medición con la escala breve de evaluación del funcionamiento y correlación con los años de enfermedad
}

\section{Schizophrenia and functioning: correlation between functioning assessment short test and years of disorder}

\author{
Miriam L. Osorio-Martínez ${ }^{1, a}$ \\ ${ }^{1}$ Servicio de Emergencia, Hospital Hermilio Valdizán, Lima, Perú. \\ ${ }^{a}$ Psiquiatra.
}

\section{Correspondencia}

Dra. Miriam L. Osorio-Martínez ml_osorio@hotmail.com

Hospital Hermilio Valdizan, DSMAG, Carretera Central km $3.5 \mathrm{~s} / \mathrm{n}$

Recibido, 29 junio 2016

Evaluado, 20 agosto 2016

Acaptado, 27 agosto 2016

Este material no ha sido publicado ni remitido a ninguna revista biomédica.

Autofinanciado.

Ningún conflicto de interes.

Citar como: Osorio-Martínez ML. Esquizofrenia y funcionamiento: medición con la escala breve de evaluación del funcionamiento y correlación con los años de enfermedad. An Fac med. 2017;78(1):17-22. DOI: http://dx.doi. org/10.15381/anales.v78i1.13016
An Fac med. 2017;78(1):17-22 / http://dx.doi.org/10.15381/anales.v78i1.13016

Resumen

Introducción. La esquizofrenia se asocia a deterioro de las capacidades de la persona, en varios ámbitos de su vida, en forma progresiva. Sin embargo, este hecho ha sido poco estudiado en nuestra población. Objetivo. Identificar la afectación en el funcionamiento según tiempo con esquizofrenia. Diseño. Estudio analítico correlacional, diseño observacional transversal. Lugar. Hospital Hermilio Valdizán, Lima, Perú. Participantes. Pacientes diagnosticados de esquizofrenia atendidos ambulatoriamente. Intervenciones. Entrevista y aplicación de escalas, a 136 pacientes, en 4 grupos de 34, según tiempo de enfermedad. El análisis se efectuó con las pruebas de t-student, Kruskall-Wallis, $U$ de Mann Whitney y Correlación de Spearman, con intervalo de confianza $95 \%, p<0,05$. Principales medidas de resultados. Medida del funcionamiento empleando la escala breve de evaluación del funcionamiento (FAST) y medida de síntomas psicóticos con escala breve de valoración de sintomas psiquiátricos (BPRS). El puntaje FAST es inversamente proporcional con el funcionamiento. Resultados. El 57\% fueron varones, $47,9 \%$ en rango de 21 a 30 años. El puntaje FAST para el grupo menor de 1 año de enfermedad fue 50,2 $\pm 9,3 \mathrm{DE}, 1$ a 5 años 57,1 $\pm 10,6 \mathrm{DE}, 6$ a 10 años 59,0 \pm 9,7 DE, y con más de 10 años de enfermedad 60,2 $\pm 10,1 \mathrm{DE}$. Se halló diferencia significativa entre el funcionamiento $(p<0,005)$ para el grupo con menos de un año de enfermedad en relación a los otros. Conclusiones. Los pacientes con menos de un año de enfermedad mostraron mejor funcionamiento. Se observó disminución del funcionamiento después del primer año de diagnóstico, indistintamente entre los grupos. Existe la necesidad de empezar estrategias de rehabilitación tempranas.

Palabras clave. Esquizofrenia, Funcionamiento; Clasificación Internacional de Funcionalidad, Discapacidad y Salud (CIF).

\section{Abstract}

Introduction: Schizophrenia is associated to progressive deterioration of the person's skills in several areas of life. However this fact has not been studied in our population. Objective: To identify the effect of schizophrenia on functioning according to time. Design: Analytical correlational, cross-sectional observational study. Location: Hospital Hermilio Valdizan, Lima, Peru. Participants: Outpatient subjects diagnosed with schizophrenia. Interventions. Interview and application of scales to 136 patients in 4 groups of 34 according to time of sickness. The analysis was performed with t-student tests, Kruskal-Wallis, Mann Whitney $U$ test and Spearman correlation, with a 95\% confidence interval, $p<0.05$. Main outcome measures: Measurement of functioning with the Short Scale Performance Review (FAST) and measurement of psychotic symptoms with the Brief Psychiatric symptoms Rating Scale (BPRS) were done. The FAST score is inversely proportional to functioning. Results: $57 \%$ of patients were male, $47.9 \%$ in the $21-30$ age range. The FAST score for the group with less than 1 year of disease was $50.2+9.3$, for $1-5$ years $57.1+10.6,6-10$ years $59.0+9.7$, and more than 10 years of illness $60.2+10.1$. There was a significant difference $(p<0.005)$ for in functioning between the group with less than one year of disease versus the other groups. Conclusions: Patients with less than one year of disease showed better functioning. Decreased functioning was observed after the first year of diagnosis, without distinction between groups. There is a need for early rehabilitation strategies starting in schizophrenia.

Keywords: Schizophrenia, Functioning; International Classification of Functioning, Disability and Health (ICF). 


\section{INTRODUCCIÓN}

La Organización Mundial de la Salud $(\mathrm{OMS})^{(1)}$ define funcionamiento como un término global, referido a las funciones corporales, actividades y participación en la vida social. Indica los aspectos positivos de la interacción entre un individuo y sus factores ambientales.

En setiembre de 2015, la OMS ${ }^{(2)}$ informó que existen 21 millones de personas en el mundo afectadas de esquizofrenia. Considerando la carga de morbilidad causada por la discapacidad, la OMS (3) atribuye a los trastornos mentales el 25,3\% y el 33,5\% de los años perdidos por discapacidad (AVAD) en los países de ingresos bajos y de ingresos medios, respectivamente.

En el estudio de Carga de Enfermedad en el Perú ${ }^{(4)}$ las enfermedades neuropsiquiátricas ocuparon el primer lugar versus los 21 grupos de daños. Ocasionaron 1010594 de años de vida saludables perdidos (AVISA), siendo el $88 \%$ de ellos por discapacidad. En el mismo estudio, la esquizofrenia representó cerca de 100 000 AVAD durante el 2012.

Frente a este panorama, la mayoría de pacientes con enfermedad mental no acude a centros especializados, y aquellos que lo hacen van a centros de salud u hospitales generales, retrasando el diagnóstico y el tratamiento oportuno.

Kohn ${ }^{(5)}$ y col, en el 2005, precisaron que se observó las psicosis no afectivas y la esquizofrenia en $1,4 \%$ (media) y $1,6 \%$ (mediana) de la población de América Latina y el Caribe, en algún momento de la vida.

Según Barrera ${ }^{(6)}$, los niveles de funcionamiento de las personas con trastornos psicóticos muestran dificultades a través de múltiples dominios, tales como la vida independiente, las relaciones interpersonales, los logros profesionales y educativos, disfrute de recreo y la actividad sexual. En el Perú, esta repercusión no ha sido evaluada en forma amplia. Estas dificultades son comunes en muchos de los pacientes, y pueden continuar por períodos prolongados dando como resultado una sostenida discapacidad, con el sufrimiento personal y familiar, y los cos- tos indirectos a la sociedad que conlleva.

El manual diagnóstico y estadístico de los trastornos mentales (DSM 5) ${ }^{(7)}$ introduce el término 'nivel de funcionamiento' como un criterio diagnóstico de la enfermedad esquizofrénica, cuando este se encuentra muy por debajo del nivel alcanzado antes del inicio de la enfermedad, o cuando no se alcanza el nivel esperado, en los menores de edad. Según Lenior ${ }^{(8)}$ y col, la completa recuperación sintomática y funcional podría ser posible solo para una minoría de los pacientes durante los primeros años de la enfermedad.

Para la evaluación del funcionamiento se fueron creando numerosas escalas, que han ido evolucionando con el tiempo. En 1974, el enfoque más amplio lo demostró la Escala Pronóstico para la Esquizofrenia de Strauss y Carpenter ${ }^{(9)}$, que realizaron el seguimiento de pacientes con esquizofrenia por cinco años y desarrollaron la escala, cuyo objetivo era predecir en forma más efectiva el pronóstico de pacientes con esquizofrenia. La escala pronóstica ha sido adaptada y validada a la versión en español por el grupo de trabajo en esquizofrenia de Barcelona, la cual hallaron bastante útil para el seguimiento de los pacientes con esquizofrenia.

Posterior a 1980 han surgido diferentes intentos de desarrollar instrumentos de evaluación global del funcionamiento, más exactas y precisas que los anteriores existentes para pacientes con trastornos mentales graves ${ }^{(10,11)}$.

La Escala Breve de Evaluación del Funcionamiento (FAST) ${ }^{(12)}$ es un instrumento diseñado para evaluar el deterioro funcional en trastornos mentales graves. Los 24 ítems de la escala cubren 6 áreas específicas de funcionamiento: la autonomía, la actividad laboral, funcionamiento cognitivo, las cuestiones financieras, relaciones interpersonales y el tiempo libre. Se trata de un instrumento sencillo, fácil de aplicar en consulta ambulatoria y requiere un tiempo corto de administración. Posee una puntuación inversa al nivel del funcionamiento: mientras mayor puntaje se alcance, mayor deterioro funcional habrá al momento de la evaluación.
El estudio de Gonzales ${ }^{(13)}$ y col., para validar la Escala Breve de Evaluación del Funcionamiento (FAST) comparándola con la Escala de Strauss-Carpenter en pacientes con primer episodio psicótico, demostró tener fuertes propiedades psicométricas, constituyéndose en un instrumento válido para su uso en la práctica clínica y ensayos clínicos, como en investigación de temas relacionados con el primer episodio psicótico.

El objetivo del presente estudio fue evaluar el funcionamiento según tiempo de enfermedad en una muestra de pacientes con esquizofrenia, atendidos en el Hospital Hermilio Valdizán de Lima, Perú.

\section{MÉTODOS}

Se realizó un estudio analítico correlacional, con el diseño observacional transversal. La población objeto de este estudio estuvo comprendida por todos los pacientes diagnosticados de esquizofrenia, que estaban en la fase de estabilización o fase estable de la enfermedad y que visitaron el servicio de consulta externa del Hospital Hermilio Valdizan durante los años 2011 y 2012.

El total de casos atendidos durante el año 2011 fue 10367 pacientes. La muestra estuvo conformada por 136 pacientes diagnosticados, los cuales fueron distribuidos en grupos de 34 . Se diseñó el consentimiento informado en forma clara y sencilla, junto con la explicación respectiva para el paciente y su familiar acompañante. Los criterios de inclusión fueron:

- Pacientes con diagnóstico de esquizofrenia, según criterios de la Clasificación Internacional de Enfermedades (CIE 10).

- Dar su consentimiento a la participación en el estudio de manera voluntaria. De no poder hacerlo, se pedía el consentimiento del apoderado.

Los criterios de exclusión fueron:

- Paciente con retraso mental.

- La no aceptación a participar en el estudio por parte del paciente o la familia. 
- Dificultades de comprensión del idioma en el caso de personas quechuahablantes.

- Diagnóstico comórbido de abuso/dependencia de sustancias psicoactivas.

La población de estudio fue clasificada en cuatro grupos: menos de un año de enfermedad, de uno a cinco años, de seis a diez años de enfermedad y más de 10 años. Los pacientes fueron evaluados mediante entrevista clínica y un protocolo que incluía las siguientes escalas: La escala breve de evaluación del funcionamiento (FAST) y la escala breve de evaluación psiquiátrica (BPRS). Se obtuvo el manual para la aplicación de la Escala Fast y el permiso de sus autores. Se solicitó la autorización del hospital para ejecutar las entrevistas y la aprobación del comité de ética para la respectiva autorización del estudio.

El procesamiento y análisis estadístico de los datos se realizó por medio del programa estadístico SPSS versión 21 . El análisis univariado de las variables sociodemográficas y clínicas se hizo a través de tablas de distribución de frecuencias, con sus respectivos gráficos de barras y sectores para las variables cualitativas. Para las variables cuantitativas, fue por medio de medias y desviación estándar.

El análisis bivariado se realizó relacionando, en tablas de contingencia, la variable funcionamiento con la variable sexo, por medio de la prueba t de student para muestras independientes. Las variables edad y años de estudio fueron categorizadas, y junto con la variable tiempo de enfermedad se relacionaron con el funcionamiento por medio de la prueba de Kruskal-Wallis. Cuando se halló diferencia estadísticamente significativa con esta prueba, se realizó la comparación pareada por medio de la prueba $U$ de Mann-Whitney. La variable ocupación se relacionó con el funcionamiento por medio de la prueba $U$ de Mann-Whitney. Para correlacionar el funcionamiento de los pacientes con los síntomas psicóticos se aplicó la correlación de Spearman. Para la elección de las pruebas antes mencionadas primero se tuvo que determinar la distribución normal de los datos por medio de las pruebas KolmogorovSmirnov y Shapiro-Wilk.
Se trabajó todas las pruebas a un nivel de significancia del 5\%, excepto la prueba de correlación, que fue trabajada al $1 \%$.

\section{RESULTADOS}

La edad al momento del diagnóstico fue 21,1 \pm 6,6 DE años, con una edad mínima de 12 y de máximo 45 años. El puntaje obtenido en la escala de BPRS fue de un mínimo de 3 y un máximo de 53, con media de $29 \pm 9,2 \mathrm{DE}$. El puntaje obtenido con la escala FAST fue de un mínimo de 29 y un máximo de 75 , con media de 56,6 \pm 10,6 DE. La tabla 1 muestra las características sociodemograficas; se observa que la edad mínima fue 15 años y la máxima 69. Los años de estudio mínimo fueron de 1 año y máximo de 20 años.

Cuando se comparó el valor del funcionamiento con sexo y ocupación (pruebas estadísticas t de student y $U$ de Mann Whitney, respectivamente) y con las variables edad y años de estudio (las cuales fueron categorizadas) por medio de la prueba de Kruskal-Wallis, se encontró relación estadísticamente significativa con todas ellas, excepto con la ocupación $(p=0,493)$. En cuanto al sexo, los varones presentaron menor valor en la escala FAST que las mujeres ( $p=0,002)$. La comparación por pares de los grupos de edad y años de estudio se realizó con la prueba U de Mann-Whitney, encontrándose que el grupo etario de 46 a 70 años presentó un mayor valor en la escala FAST respecto a los otros grupos $(p<0,001)$, mientras que el nivel de estudios primario mostró un mayor valor en la escala FAST que los otros niveles $(p<0,05)$ (tabla 2$)$.

Se estableció la correlación entre el tiempo de enfermedad y el valor del funcionamiento, entre los cuatro grupos etarios. Se observó diferencia significativa en el grupo de menos de 1 año de enfermedad $(p<0,001)$; luego se realizó la comparación pareada por medio de la prueba U de Mann-Whitney (tabla 3).

Al evaluar la diferencia significativa entre los síntomas psicóticos y el valor de funcionamiento, se halló un $p<0,001$. Se realizó la prueba de correlación de rangos de Spearman, obteniéndose el valor de $r s=0,388$ (figura 2).

Tabla 1. Características sociodemográficas de los pacientes del estudio $(n=136)$.

\begin{tabular}{|c|c|c|}
\hline Características sociodemográficas & $\mathrm{n}$ & $\%$ \\
\hline \multicolumn{3}{|l|}{ Sexo } \\
\hline Masculino & 79 & 58,1 \\
\hline Femenino & 57 & 41,9 \\
\hline \multicolumn{3}{|l|}{ Edad } \\
\hline Media +DE* & \multicolumn{2}{|c|}{$29,7+11,4$} \\
\hline \multicolumn{3}{|l|}{ Estado civil } \\
\hline Soltero & 126 & 92,6 \\
\hline Casado/conviviente & 10 & 7,4 \\
\hline Separado/divorciado & 0 & 0 \\
\hline Viudo & 0 & 0 \\
\hline \multicolumn{3}{|l|}{ Años de estudio } \\
\hline Media +DE* & \multicolumn{2}{|c|}{$11,0+2,6$} \\
\hline \multicolumn{3}{|l|}{ Ocupación } \\
\hline Con ocupación & 70 & 51,5 \\
\hline Sin ocupación & 66 & 48,5 \\
\hline
\end{tabular}


Tabla 2. Comparación del valor del funcionamiento con las variables sexo, edad, años de estudio y ocupación.

\begin{tabular}{|c|c|c|c|c|}
\hline \multirow[b]{2}{*}{ Grupo } & \multirow[b]{2}{*}{$\mathrm{n}$} & \multicolumn{2}{|c|}{ Funcionamiento } & \multirow[b]{2}{*}{ Valor $p$} \\
\hline & & Media & $\mathrm{DE}^{*}$ & \\
\hline \multicolumn{5}{|l|}{ Sexo } \\
\hline Masculino & 79 & 54,2 & 10,7 & \multirow[t]{2}{*}{0,002} \\
\hline Femenino & 57 & 59,9 & 9,5 & \\
\hline \multicolumn{5}{|l|}{ Edad } \\
\hline 15 a 30 años & 93 & $55,5^{a}$ & 10,5 & \multirow[t]{3}{*}{0,003} \\
\hline 31 a 45 años & 26 & $55,7^{\mathrm{a}}$ & 8,5 & \\
\hline 46 a 70 años & 17 & $63,8^{b}$ & 11,8 & \\
\hline \multicolumn{5}{|l|}{ Años de estudio } \\
\hline Primaria & 9 & $63,6^{\mathrm{a}}$ & 10,2 & \multirow[t]{3}{*}{0,013} \\
\hline Secundaria & 88 & $57,6^{b}$ & 9,2 & \\
\hline Superior & 39 & $52,8^{b}$ & 12,5 & \\
\hline \multicolumn{5}{|l|}{ Ocupación } \\
\hline Con ocupación & 70 & 55,8 & 11,4 & \multirow[t]{2}{*}{0,493} \\
\hline Sin ocupación & 66 & 57,4 & 9,7 & \\
\hline
\end{tabular}

* $\mathrm{DE}=$ Desviación estándar.

† Se aplicó las pruebas, t de student, Kruskal-Wallis y $U$ de Mann-Whitney.

Diferentes letras indican diferencias estadistcamente significativas $(p<0,05)$.

Tabla 3. Correlación entre tiempo de enfermedad y puntaje obtenido con la escala FAST.

\begin{tabular}{ccccc} 
Tiempo de & & \multicolumn{2}{c}{ Funcionamiento } & \\
\cline { 3 - 4 } enfermedad & $\mathrm{n}$ & Media & $\mathrm{DE}^{*}$ & Valor $\mathrm{p}^{\dagger}$ \\
\hline < 1 año & 34 & 50,2 & 9,3 & $<0,001$ \\
1 a 5 años & 34 & 57,1 & 10,6 & \\
6 a 10 años & 34 & 59,0 & 9,7 & \\
$>10$ años & 34 & 60,2 & 10,1 & \\
\hline
\end{tabular}

* $\mathrm{DE}=$ Desviación estándar.

† Prueba de Kruskal-Wallis.

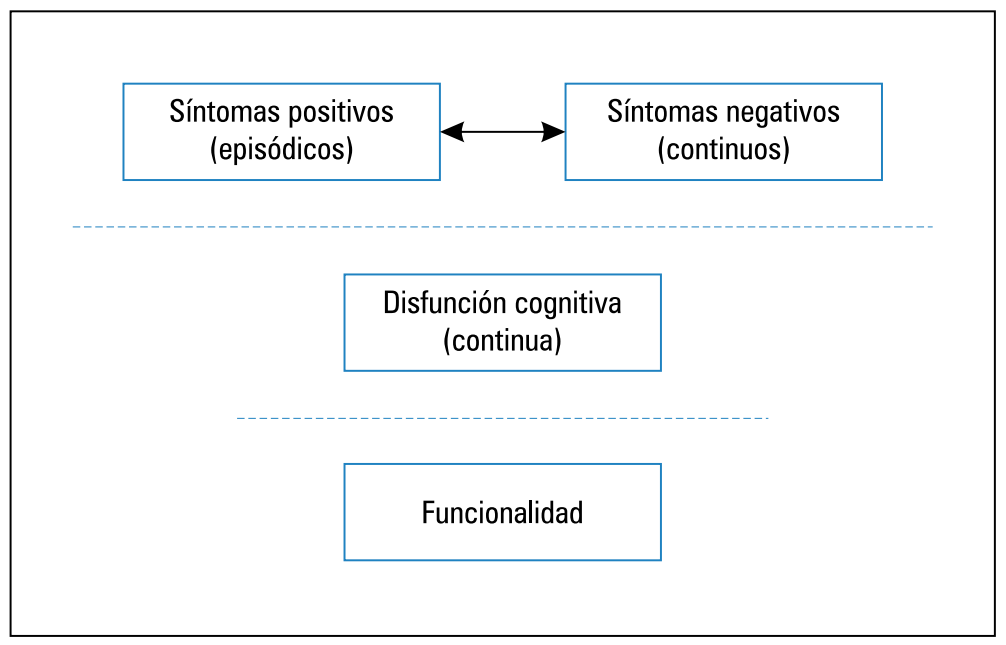

Figura 1. Complejo de relaciones entre los síntomas, cognición y funcionalidad en la esquizofrenia (tomado de Ciudad y col 2011).

\section{DISCUSION}

Se propuso como objetivo determinar la progresión del deterioro funcional según los años de la enfermedad, medidos a través de un instrumento de fácil aplicación en la práctica ambulatoria. En la muestra se obtuvo mayor porcentaje de varones. La edad media estuvo en 29,7+11,4 años, correspondiente a una población de adultos jóvenes. Los años de escolaridad en promedio fueron $11,0+2,6$ años de estudio, es decir, la mayoría había cursado o cursaba estudios secundarios. Para la evaluación de los valores obtenidos mediante la escala FAST, se encontró que fueron los varones que obtuvieron mejor nivel de funcionamiento comparado con las mujeres del estudio. Este hecho se encuentra en discusión; los diferentes resultados en estudios no señalan una diferencia estadísticamente significativa entre el funcionamiento de hombres y mujeres, siendo aun controversial este tema ${ }^{(14)}$.

Se encuentra relación directa entre el tiempo de enfermedad y el puntaje de funcionalidad medido a través de la escala FAST. Es decir, a mayor tiempo de enfermedad mayor puntaje FAST y, por lo tanto, menor grado de funcionamiento. En nuestro estudio hallamos una diferencia marcada entre el grado de funcionamiento en pacientes menores y hasta un año de enfermedad, comparado con los otros grupos. Se observó que durante el primer año de la enfermedad la persona afectada mantuvo mejor funcionamiento en las 6 esferas de evaluación de la escala FAST (autonomía, actividad laboral, funcionamiento cognitivo, cuestiones financieras, relaciones interpersonales y tiempo libre). La presencia de síntomas psicóticos medidos a través de la escala BPRS, no afectaron los resultados obtenidos al medir la funcionalidad.

Según lo reportado por Keefe ${ }^{(15)}$ y col., casi todos los pacientes con esquizofrenia muestran en alguna medida una declinación del nivel esperado de función neurocognitiva, repercutiendo en su desenvolvimiento personal, social y familiar. Green ${ }^{(16)}$ y col han observado que las fallas neurocognitivas son una característica nuclear en la esquizofrenia y no solo un resultado de los síntomas o el tratamiento utilizado para la enfermedad. 


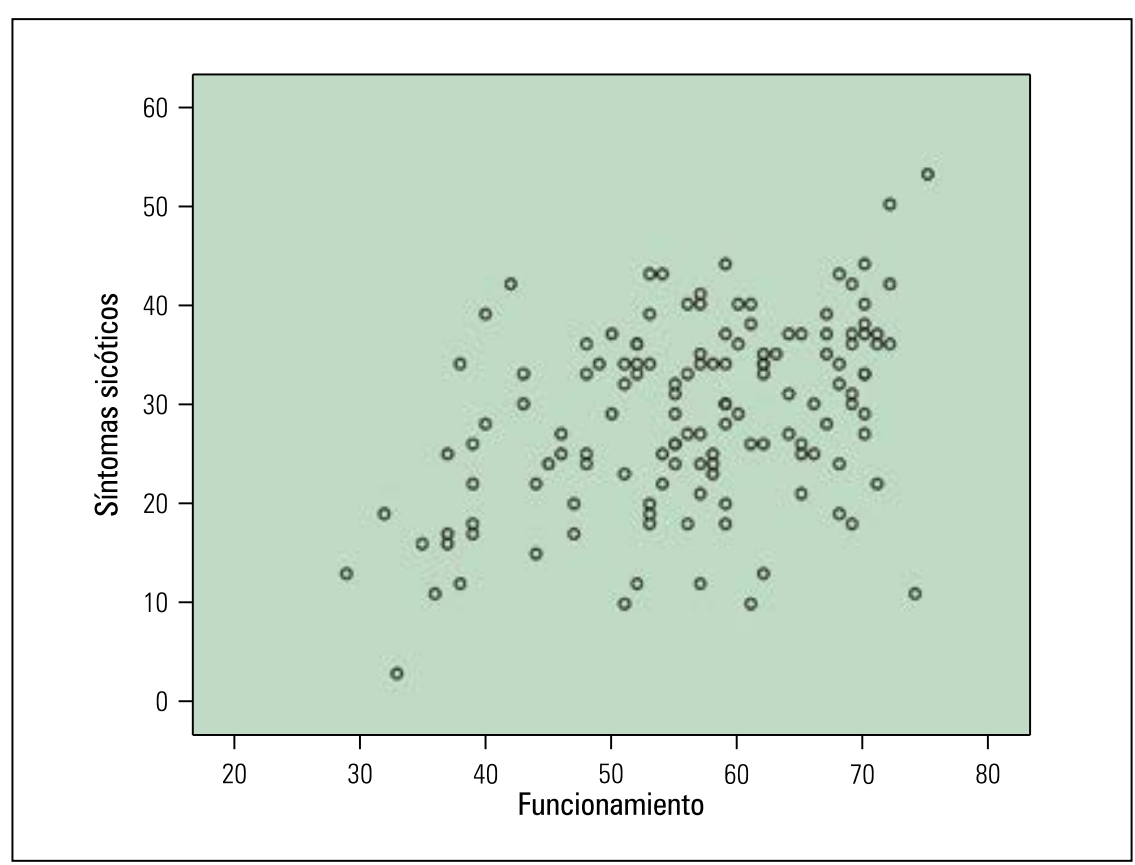

Figura 2. Correlación entre síntomas psicóticos y funcionamiento y coeficiente de Spearman $(N=136, p<0,001$. Rs $=0,388)$.

Para lo cual tomamos el gráfico (figura 1) realizado por Ciudad ${ }^{(17)}$ y col., donde observamos los diferentes componentes de la enfermedad y, por ende, la afectación en la funcionalidad como un continuum en la vida de la persona afectada.

Según Zortéa ${ }^{(18)}$ y col., la escala Fast demostró buenas propiedades psicométricas en términos de validez y confiabilidad, al ser usada en pacientes con el diagnóstico de esquizofrenia. Durante la realización del estudio, la escala FAST fue de fácil aplicación y tabulación. Por tanto se la sugiere como instrumento útil para obtener información de la discapacidad en estos pacientes, en el control ambulatorio.

Los resultados obtenidos concuerdan con lo reportado en la literatura para la esquizofrenia. Se muestra progresión continua durante la vida de la persona afectada y la separación en el rendimiento funcional entre los pacientes con esquizofrenia menores de un año de enfermedad y aquellos de mayor tiempo de enfermedad. Estas fallas del funcionamiento en el mundo real están fuertemente correlacionadas al déficit neurocognitivo, según lo reportado por Green ${ }^{(19)}$.
Nuestro estudio se realizó en un hospital nacional con gran afluencia de pacientes de todas partes del Perú. Se hizo la búsqueda en la literatura de material relacionado al funcionamiento, en los términos planteados por la OMS, no encontrándose resultados al respecto. Por lo tanto, se observa la necesidad de poder replicar el estudio en otras sedes de tratamiento de enfermedades mentales y poder establecer estadiaje de la funcionalidad en la esquizofrenia a nivel del Perú. La identificación temprana del deterioro en el funcionamiento beneficiará las estrategias de rehabilitación dirigidas a la población afectada.

\section{AGRADECIMIENTOS}

A las personas que sufren de enfermedades mentales y a sus familias, que colaboraron en el estudio. Al Dr. César Gutiérrez V., por su experticia y paciencia en la guía para la realización del estudio. A la Dra. Adriane Rosa y el Dr. Eduard Vieta, por su ayuda desinteresada con sus opiniones y el permiso para el uso de la Escala FAST.

\section{REFERENCIAS BIBLIOGRÁFICAS}

1. Ayuso-Mateos JL, Nieto-Moreno M, Sánchez-Moreno J, Vázquez-Barquero JL. Clasificación internacional del funcionamiento, la discapacidad y la salud (CIF): aplicabilidad y utilidad en la práctica clínica. Med Clin (Barc) [Internet] 2006; [citado 2011 Jul 15;126(12):461-6]. doi: 10.1157/13086326.

2. Organización Mundial de la Salud [Internet]; Ginebra, OMS. Nota descriptiva $N^{\circ}$ 397. 2015. [citado 2013 Feb 11]; Disponible en: http://www.who.int/ mediacentre/factsheets/fs397/es/

3. Organización Mundial de la Salud. Carga mundial de trastornos mentales y necesidad de que el sector de la salud y el sector social respondan de modo integral y coordinado a escala de país. EB 130/9. 2011. [citado 2012 Mar 15]; Disponible en: http://apps.who.int/gb/ebwha/pdf_files/EB130/ B130_9-sp.pdf

4. Ministerio de Salud. Estudio de Carga de Enfermedad en el Perú. Estimación de los Años de Vida Saludables Perdidos 2012. Lima. Dirección General de Epidemiologia. 2014.

5. Kohn R, Levav I, Almeida JM, Vicente B, Andrade L, Caraveo-Anduaga JJ y col. Los trastornos mentales en América Latina y el Caribe: asunto prioritario para la salud pública. Rev Panam Salud Pública [Internet] 2005 Nov [citado 2014 Feb 17]; 18( 4-5 ): 229-240. Disponible: http://dx.doi.org/10.1590/ S1020-49892005000900002.

6. Barrera PA. Los trastornos cognitivos de la esquizofrenia. Rev chil neuro-psiquiatr [Internet] 2006 [citado 2013 Jun 17];44(3):215-21.Disponible: http://www.scielo.cl/scielo.php?script=sci_ arttext\&pid=S0717-92272006000300007\&Ing=es.

7. Asociación Psiquiátrica Americana. Sección II: Espectro de la esquizofrenia y otros trastornos psicóticos. En: Manual Diagnostico y Estadistico de las enfermedades Mentales 5. 5ta edición. Argentina. Editorial Médica Panamericana. 2014.

8. Lenior ME, Dingemans P, Schene AH, Linszen DH. Predictors of the early 5-year course of schizophrenia: a path analysis. Schizophr Bull [Internet] 2005 [Citado 2013 Feb 17]; 31 (3):781-791. Disponible en http://schizophreniabulletin.oxfordjournals.org/ content/31/3/781.full.

9. Rosa AR, Sánchez-Moreno J, Martínez-Aran A, Salamero M, Torrent C, Reinares M, et al. Validity and reliability of the Functioning Assessment Short Test (FAST) in bipolar disorder. Clin Pract Epidemol Ment Health [Internet] 2007[citado 2013 Abr 12]; 3:5. Disponible en: http://download.springer.com/static/pdf/10/art\%253A10. 1186\%252F1745-0179-3-5.pdf?originUrl=http $\% 3 \mathrm{~A} \% 2 \mathrm{~F} \% 2 \mathrm{Fcpementalhealth}$. biomedcentral. com\%2Farticle\%2F10.1186\%2F1745-0179-35 \&token2 $=\exp =1459789560 \sim$ acl $=\% 2 F$ static $\%$ 2Fpdf\%2F10\%2Fart\%25253A10.1186\%2525 2F1745-0179-3-5.pdf* $\sim$ hmac $=$ b238d0e9c08494 $5 \mathrm{ffa} 277 \mathrm{eb0fc084e2e289d947620cce25668695b-}$ $31 \mathrm{~d} 7829 a 22$

10. Kleinman L, Lieberman J, Dube S, Mohs R, Zhao $Y$, Kinon B, et al. Development and psychometric performance of the schizophrenia objective functioning instrument: An interviewer administered measure of function. Schizophr Res. [internet] 2009 [citado 2011 Nov 1] Feb 1;107 (2-3):275-285. doi: 10.1016/j.schres.2008.10.002

11. Chen L, Phillips G, Johnston J, Kinon BJ, AscherSvanum H, Kollack-Walker S, et al. The relationship, structure and profiles of schizophrenia 
measurements: a post-hoc analysis of the baseline measures from a randomized clinical trial. BMC Psychiatry. [Internet] 2011 [citado 2012 En 12]; 11(1), 203. doi: 10.1186/1471-244X-11-203

12. Ribeiro RA, Sanchez-Moreno J, Martinez- Aran A Godelieve W, Bonnin C, Ayuso-Mateos JL y col. Prueba Breve de Evaluacion del Funcionamiento (FAST) [internet] 2008 [citado 2013 Jul 12]; en: http://bi.cibersam.es/media/1003/guia_de_administracion.pdf.

13. González-Ortega I, Rosa A, Alberich S, Barbeito $S$, Vega $P$, Echeburúa $E$, et al. A validation and use of the functioning assessment short test in first psychotic episodes [internet]. J Nerv Ment Dis. 2010 [citado 2013 Jun 6]. 198(11):836-40. Doi: 10.1097/NMD. 0b013e3181f97bf9

14. Ochoa S, Usall J, Cobo J, Labad X, Kulkarni J. Gender differences in schizophrenia and firstepisode psychosis: a comprehensive literature review. Schizophr Res Treatment. [Internet] 2012 [citado 2016 Jun 12] Article ID 916198. Doi: 10.1155/2012/916198.

15. Keefe RSE, Eesley CE, Poe MP. Defining a cognitive function decrement in schizophrenia [internet] Biol Psychiatr. 2005 [citado 2013 May 23]. 57: 688-691. Doi: 10.1016/j.biopsych.2005.01.003.
16. Green MF, Nuechterlein KH, Gold JM, Barch D, Cohen J, Essock S, et al. Approaching a consensus cognitive battery for clinical trials in schizophrenia: the NIMH-MATRICS conference to select cognitive domains and test criteria [internet] Biol Psychiatry. 2004 [citado 2013 Oct 8]. 56: 301-307. Disponible en http://dx.doi.org/10.1016/j. biopsych.2004.06.023.

17. Ciudad A, Bobes J, Álvarez E, San L, Novick D, Gilaberte I. Resultados clínicos relevantes en esquizofrenia: remisión y recuperación [internet]. Rev Psiquiatr Salud Ment. 2011 [citado 2012 Jul 16]; 4(1), 53-65. Doi: 10.1016/j.rpsm.2010.12.002.

18. Zortéa K, Da Silva P, Rosa AR, De Lucena D, Guimarães LR, Francesconi LPP, et al. Concurrent validity and reliability of the Brazilian version of the functioning assessment short test in patients with schizophrenia. Value Health Reg Issues. [internet] 2012 [citado 2013 Jun 11] 1(2), 244-247. Disponible en: http://www.valuehealthregionalissues.com/ article/S2212-1099(12)00053-2/pdf.

19. Green MF. What are the functional consequences of neurocognitive deficits in schizophrenia? Am J Psychiatry. [internet] 1996 [citado 2012 Jun 15] 153:321-30. Disponible en: http://ajp.psychiatryonline.org/doi/pdf/10.1176/ajp.153.3.321. 